\title{
On Planar Polyline Drawings
}

\author{
Huaming Zhang and Sadish Sadasivam \\ Computer Science Department \\ University of Alabama in Huntsville \\ Huntsville, AL, 35899, USA \\ $\{$ hzhang, ssadasiv\}@cs.uah.edu
}

\begin{abstract}
We present a linear time algorithm that produces a planar polyline drawing for a plane graph with $n$ vertices in a grid of size bounded by $(p+1) \times(n-2)$, where $p \leq\left(\left\lfloor\frac{2 n-5}{3}\right\rfloor\right)$. It uses at most $p \leq\left\lfloor\frac{2 n-5}{3}\right\rfloor$ bends, and each edge uses at most one bend. Compared with the area optimal polyline drawing algorithm in [3], our algorithm uses a larger grid size bound in trade for a smaller bound on the total number of bends. Their bend bound is $(n-2)$. Our algorithm is based on a transformation from Schnyder's realizers 6]7] of maximal plane graphs to transversal structures [4/5] for maximal internally 4-connected plane graphs. This transformation reveals important relations between the two combinatorial structures for plane graphs, which is of independent interest.
\end{abstract}

\section{Introduction}

We focus on planar graph drawings. Such graphs can be drawn without any edge crossings. Several styles of drawings [1 have been introduced. Common objectives include small area, few bends and good angular resolution. We deal with polyline drawings [1. A polyline drawing is a drawing of a graph in which each edge is represented by a polygonal chain and every vertex is placed on a grid point. Bonichon et al. 3] presented a linear time algorithm that produces polyline drawings for a graph with $n$ vertices within a grid of area $\left(n-\left\lfloor\frac{p}{2}\right\rfloor-1\right) \times(p+1)$, where $p \leq \frac{2 n-5}{3}$. It is area optimal and each edge has at most one bend. However the total number of bends used by this algorithm could be $(n-2)$.

Our goal is to have a tradeoff between the grid size and the number of bends. We present a linear time algorithm that produces a polyline drawing in a grid with size bounded by $(p+1) \times(n-2)$, where $p \leq\left\lfloor\frac{2 n-5}{3}\right\rfloor$, and each edge uses at most one bend. Although the grid size is not as good as the algorithm in [3], our algorithm only needs at most $p \leq\left\lfloor\frac{2 n-5}{3}\right\rfloor$ bends.

A maximal plane graph $G$ is associated with realizers $\mathcal{R}$ [67, which is a partition of the set of interior edges into three particular trees. A maximal internally 4-connected plane graph $G^{\prime}$ with four exterior vertices is associated with transversal structures $\mathcal{T}$ [45], which is a partition of the set of interior edges into two st-graphs. In this paper, we introduce a transformation from a maximal plane graph $G$ to a maximal internally 4-connected plane graph $G^{\prime}$ with 
four exterior vertices by a certain number of operations. These operations are determined by a realizer of $G$ and can be done in linear time. Then our algorithm uses the derived $G^{\prime}$ and its transversal structure to obtain the polyline drawing.

The present paper is organized as follows. In Section 2 we recall a few definitions. In Section 3, we present the transformation from a realizer to a transversal structure. Then we present our drawing algorithm.

\section{Preliminaries}

The graphs are simple graphs. We abbreviate "counter clockwise" and "clockwise" as ccw and cw respectively.

Definition 1. [6] Let $G$ be a maximal plane graph of $n$ vertices with three exterior vertices $v_{1}, v_{2}, v_{3}$ in ccw order. A realizer $\mathcal{R}(G)=\left\{T_{1}, T_{2}, T_{3}\right\}$ of $G$ is a partition of its interior edges into three sets $T_{1}, T_{2}, T_{3}$ of directed edges such that the following holds: (1) for each $i \in\{1,2,3\}$, the interior edges incident to $v_{i}$ are in $T_{i}$ and directed toward $v_{i}$; (2) for each interior vertex of $G$, $v$ has exactly one edge leaving $v$ in each of $T_{1}, T_{2}, T_{3}$. The ccw order of the edges incident to $v$ is: leaving in $T_{1}$, entering in $T_{3}$, leaving in $T_{2}$, entering in $T_{1}$, leaving in $T_{3}$ and entering in $T_{2}$. Each entering block could be empty.

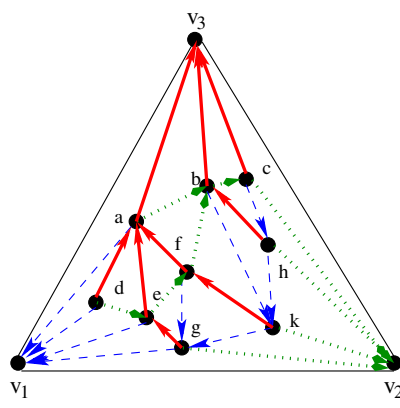

(1)

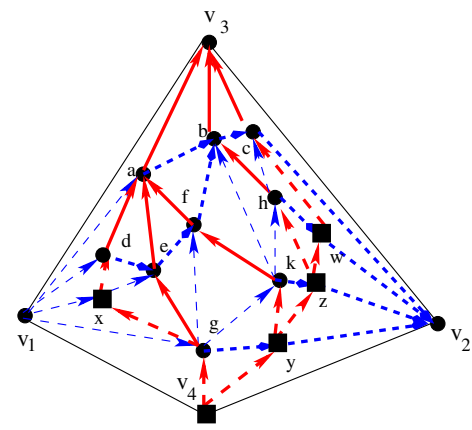

(2)

Fig. 1. (1) A maximal plane graph $G$ and a realizer $\mathcal{R}(G)$ of $G$. (2) A maximal internally 4-connected plane graph $G^{\prime}$ with four exterior vertices and a transversal structure $\mathcal{T}\left(G^{\prime}\right)$ for $G^{\prime}$.

Schnyder presented a linear time algorithm to construct a realizer for $G$. An example of a maximal plane graph $G$, and one of its realizers is given in Fig. 1 . (1). Next, we introduce the concept of transversal structures [45].

Definition 2. let $G^{\prime}$ be a maximal internally 4-connected plane graph with four exterior vertices $v_{1}, v_{4}, v_{2}$, and $v_{3}$ in ccw order. $A$ transversal structure $\mathcal{T}\left(G^{\prime}\right)$ of $G^{\prime}$ is a partition of its interior edges into two sets, say in red and blue edges, such that the following conditions are satisfied: 
1. In cw order around each interior vertex $v$, its incident edges form: a non empty interval of red edges entering $v$, a non empty interval of blue edges entering $v$, a non empty interval of red edges leaving $v$, and a non empty empty interval of blue edges leaving $v$.

2. All interior edges incident to $v_{3}$ are red edges entering $v_{3}$, all interior edges incident to $v_{4}$ are red edges leaving $v_{4}$, all interior edges incident to $v_{1}$ are blue edges leaving $v_{1}$, and all interior edges incident to $v_{2}$ are blue edges entering $v_{2}$. Each such block is non empty.

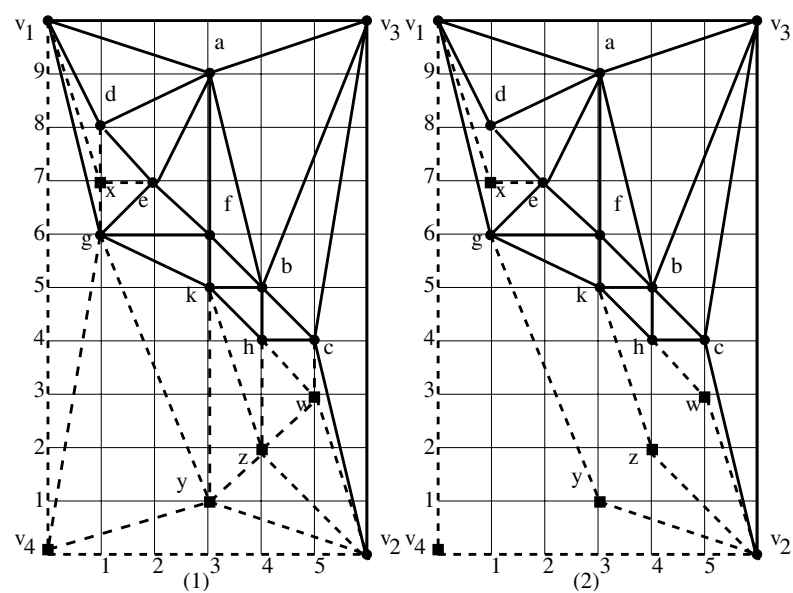

Fig. 2. (1) A straight-line grid drawing of the graph $G^{\prime}$ in Fig. 11(2). (2) A polyline drawing of $G$ in Fig. 1 (1).

Fig. 1 (2) shows an example of a transversal structure $\mathcal{T}\left(G^{\prime}\right)$ for a maximal internally 4-connected plane graph $G^{\prime}$ with four exterior vertices. The subgraph of $G^{\prime}$ with all its red-colored edges (blue colored edges respectively) and all its four exterior edges is called a red map (blue map respectively) of $G^{\prime}$, it is denoted by $G_{r}^{\prime}\left(G_{b}^{\prime}\right.$ respectively). For any interior vertex $v$ of $G^{\prime}$, let $P_{b}(v)$ be the unique path from $v_{1}$ to $v_{2}$ in $G_{b}^{\prime}$ such that, the subpath of $P_{b}(v)$ from $v_{1}$ to $v$ is the rightmost one before arriving at $v$, and the subpath of $P_{b}(v)$ from $v$ to $v_{2}$ is the leftmost one after leaving $v$. Let $y(v)$ be the number of faces in $G_{b}^{\prime}$ enclosed by the path $\left(v_{1}, v_{4}, v_{2}\right)$ and $P_{b}(v)$. Similarly, for any interior vertex $v$ of $G^{\prime}$, let $P_{r}(v)$ be the unique path from $v_{4}$ to $v_{3}$ in $G_{r}^{\prime}$ such that, the subpath of $P_{r}(v)$ from $v_{4}$ to $v$ is the rightmost one before arriving at $v$, and the subpath of $P_{r}(v)$ from $v$ to $v_{3}$ is the leftmost one after leaving $v$. Let $x(v)$ be the number of faces in $G_{r}^{\prime}$ enclosed by the path $\left(v_{4}, v_{1}, v_{3}\right)$ and $P_{r}(v)$.For example, vertex $k$ in Fig. 1 (2) satisfies $P_{r}(k)=\left(v_{4}, y, k, f, a, v_{3}\right)$, so that $x(k)=3$; and $P_{b}(k)=\left(v_{1}, g, k, b, c, v_{2}\right)$, so that $y(k)=5$. Let $x\left(\mathcal{T}\left(G^{\prime}\right)\right)$ be the number of interior faces of $G_{r}^{\prime} \cdot y\left(\mathcal{T}\left(G^{\prime}\right)\right)$ be the number of interior faces of $G_{b}^{\prime}$. For the vertices $v_{1}, v_{2}, v_{3}, v_{4}$, we define $x\left(v_{1}\right)=0, y\left(v_{1}\right)=y\left(\mathcal{T}\left(G^{\prime}\right)\right), x\left(v_{4}\right)=0, y\left(v_{4}\right)=0$, 
$x\left(v_{3}\right)=x\left(\mathcal{T}\left(G^{\prime}\right)\right), y\left(v_{3}\right)=y\left(\mathcal{T}\left(G^{\prime}\right)\right)$, and $x\left(v_{2}\right)=x\left(\mathcal{T}\left(G^{\prime}\right)\right), y\left(v_{2}\right)=0$. We have the following lemma from [4]:

Lemma 1. Let $G^{\prime}$ be a maximal internally 4-connected plane graph with 4 exterior vertices $v_{1}, v_{4}, v_{2}, v_{3}$ in ccw order. Then:

1. $G^{\prime}$ admits a transversal structure $\mathcal{T}\left(G^{\prime}\right)$, which is computable in linear time.

2. Applying $\mathcal{T}\left(G^{\prime}\right)$, for each vertex $v$, embed it in the grid point $(x(v), y(v))$. For each edge of $G^{\prime}$, simply connect its end vertices by a straight line. The drawing is a straight-line grid drawing for $G^{\prime}$. Its drawing size is $x\left(\mathcal{T}\left(G^{\prime}\right)\right) \times$ $y\left(\mathcal{T}\left(G^{\prime}\right)\right)$. This drawing is computable in linear time.

Fig. 2 (1) presents a straight-line grid drawing of the graph of $G^{\prime}$ in Fig. 1 (2), by applying Lemma 1 to $\mathcal{T}\left(G^{\prime}\right)$ in Fig. 1 (2).

\section{Transformation from Realizers to Transversal Structures and Its Application in Planar Polyline Drawing}

Let $G$ be a maximal plane graph with 3 exterior vertices $v_{1}, v_{2}, v_{3}$ in ccw order. Let $\mathcal{R}(G)=\left\{T_{1}, T_{2}, T_{3}\right\}$ be one of its realizers. $T_{i}$ is rooted at $v_{i}$. Next, we illustrate how to transform a realizer for $G$ to a transversal structure for a targeted maximal internally 4-connected plane graph $G^{\prime}$ with 4 exterior vertices. Our transformation uses a tree from $\mathcal{R}(G)$. Subject to a color and index rotation, we only need to show the case of using $T_{3}$. Let $v$ be a leaf node of $T_{3} . v$ is an interior vertex of $G$. Let $p_{1}(v)$ and $p_{2}(v)$ be its parents in $T_{1}$ and $T_{2}$ respectively. The face $f$ enclosed by $\left\{v, p_{1}(v), p_{2}(v)\right\}$ is an interior face of $G$. Consider the edge $e=\left(p_{1}(v), p_{2}(v)\right)$. According to the property of realizer, $e$ cannot be in $T_{3}$. Furthermore, e cannot be $\left(v_{1}, v_{3}\right)$, neither can it be $\left(v_{2}, v_{3}\right)$.

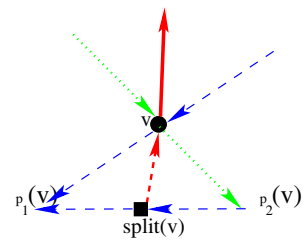

(1)

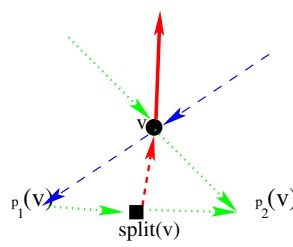

(2)

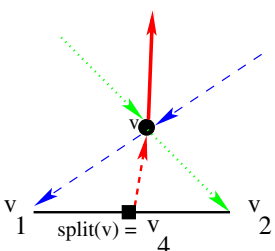

(3)

Fig. 3. Step 1

We complete the transformation in the following three steps. We will use $G^{\prime}$ to denote both the target graph and the intermediate forms.

Step 1: For every leaf node $v$ of $T_{3}$, insert a vertex split $(v)$ in the middle of $e=\left(p_{1}(v), p_{2}(v)\right)$. split $(v)$ splits $e$ into two edges. Let the two edges keep the original color and directions as $e$ in $G$. Add a directed edge from $\operatorname{split}(v)$ to $v$, 
and color it by red. We have three different cases, as illustrated in (1), (2) and (3) of Fig. 3. Note that, in Fig. 3 (3), for the case where $e=\left(v_{1}, v_{2}\right)$, we denote the inserted vertex by $v_{4} . v_{4}$ is an exterior vertex of $G^{\prime} . G^{\prime}$ has 4 exterior vertices $v_{1}, v_{4}, v_{2}, v_{3}$ in ccw order.

Step 2: For each leaf $v$ of $T_{3}$, still consider the edge $e=\left(p_{1}(v), p_{2}(v)\right)$, as if it were not split. There are three cases to consider:

Case 1: $e=\left(v_{1}, v_{2}\right)$. No additional operation needed.

Case 2: $e$ is in $T_{1}$. $e$ is adjacent to another triangle $g$. Let $u$ be the vertex $\notin\left\{p_{1}(v), p_{2}(v)\right\}$ in $g$. According to the properties of realizer, only five scenarios are possible. They are shown in Fig. 4. In Fig. 4 (1) or (2), we add a directed edge from $u$ to $\operatorname{split}(v)$, and color it by red. In Fig. 4 (3) or (4), consider $p_{2}(v)$, it must also be a leaf in $T_{3}$. Therefore, in Step 1, a vertex $\operatorname{split}\left(p_{2}(v)\right)$ and an edge $\left(\operatorname{split}\left(p_{2}(v)\right), p_{2}(v)\right)$ have been inserted for it already. In this step, we further add an edge, directed from $\operatorname{split}\left(p_{2}(v)\right)$ to $\operatorname{split}(v)$, and color it by red. Fig. 4 (5) is similar to Fig. 4 (3) or (4) except that $u=v_{2}$.

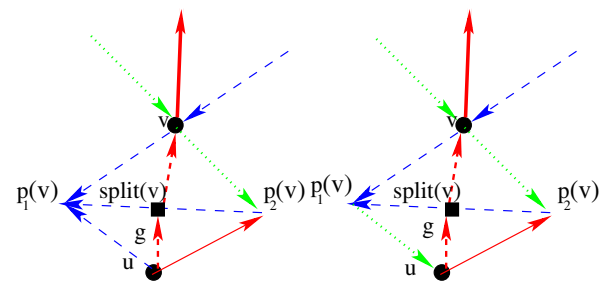

(1)

(2)

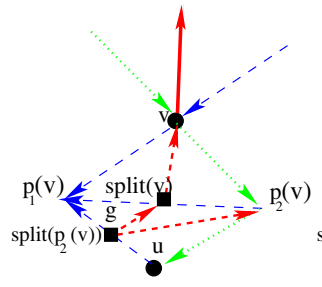

(3)

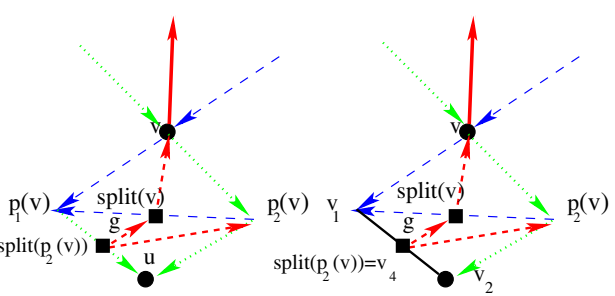

(4)

(5)

Fig. 4. Case 2 of Step 2

Case 3: $e$ is in $T_{2}$. This case is similar to Case 2.

Step 3: Reverse the direction of the blue-colored edges in $G^{\prime}$. Recolor the green-colored edges by blue.

The above coloring and directions of the edges of $G^{\prime}$ is denoted by $\mathcal{T}_{3}\left(G^{\prime}\right)$. (If we use $T_{1}, T_{2}$ instead, then we denote it by $\mathcal{T}_{1}\left(G^{\prime}\right), \mathcal{T}_{2}\left(G^{\prime}\right)$ instead). The proof of the following lemma is omitted here due to space limitation.

Lemma 2. Let $G$ be a maximal plane graph with $n$ vertices. $v_{1}, v_{2}, v_{3}$ be its exterior vertices in ccw order. $\mathcal{R}(G)=\left\{T_{1}, T_{2}, T_{3}\right\}$ be one of its realizers. $T_{i}$ is 
rooted at $v_{i}$. Let $l_{i}$ be the number of leaves of $T_{i}, i \in\{1,2,3\}$. Then for the above introduced transformation:

1. $\mathcal{T}_{i}\left(G^{\prime}\right)$ is a transversal structure of $G^{\prime} . x\left(\mathcal{T}_{i}\left(G^{\prime}\right)\right)=l_{i}+1$ and $y\left(\mathcal{T}_{i}\left(G^{\prime}\right)\right)=$ $(n-2)$, where $i \in\{1,2,3\}$.

2. The transformation from the realizer $\mathcal{R}(G)$ to $\mathcal{T}_{i}\left(G^{\prime}\right), i \in\{1,2,3\}$ can be done in linear time.

For the maximal plane graph $G$ in Fig. 1(1), Fig.11(2) shows a transversal structure $\mathcal{T}_{3}\left(G^{\prime}\right)$, constructed as above by using $T_{3}$ in the realizer $\mathcal{R}(G)=\left\{T_{1}, T_{2}, T_{3}\right\}$. The inserted vertices are represented by black squares. The inserted red-colored edges are drawn in dashed lines.

Applying Lemma 1 to $\mathcal{T}_{i}\left(G^{\prime}\right)$, we obtain a straight-line grid drawing of $G^{\prime}$. By removing the inserted edges and the inserted vertices, but keeping the split edges in the drawing of $G^{\prime}$, it becomes a polyline drawing of $G$. It is easy to see that, only an edge in $G$ which has had a vertex inserted in it during the transformation maybe drawn as two-segment polylines. The total number of such edges is $l_{i}$, i.e., the number of leaves in $T_{i}$. In [2, Bonichon et al. proved that in any realizer, $l_{1}+l_{2}+l_{3} \leq(2 n-5)$. Combined with Lemma 2] we have the following theorem:

Theorem 1. A plane graph $G$ with $n$ vertices admits a polyline drawing in a grid with size bounded by $(p+1) \times(n-2)$, where $p \leq\left\lfloor\frac{2 n-5}{3}\right\rfloor$. The number of bends is at most $p$, and each edge has at most one bend. The drawing can be constructed in linear time.

Fig. 2 (2) shows a polyline drawing of the original graph $G$ in Fig. 1 (1), where the edges represented as two-segment polylines are drawn in dashed lines.

\section{References}

1. di Battista, G., Eades, P., Tamassia, R., Tollis, I.: Graph Drawing: Algorithms for the Visualization of Graphs. Prentice Hall, Englewood Cliffs (1998)

2. Bonichon, N., Le Saëc, B., Mosbah, M.: Wagner's theorem on realizers. In: Widmayer, P., Triguero, F., Morales, R., Hennessy, M., Eidenbenz, S., Conejo, R. (eds.) ICALP 2002. LNCS, vol. 2380, pp. 1043-1053. Springer, Heidelberg (2002)

3. Bonichon, N., Le Saëc, B., Mosbah, M.: Optimal area algorithm for planar polyline drawings. In: Kučera, L. (ed.) WG 2002. LNCS, vol. 2573, pp. 35-46. Springer, Heidelberg (2002)

4. Fusy, É.: Transversal structures on triangulations, with application to straight-line drawing. In: Healy, P., Nikolov, N.S. (eds.) GD 2005. LNCS, vol. 3843, pp. 177-188. Springer, Heidelberg (2006)

5. He, X.: On finding the rectangular duals of planar triangular graphs. SIAM Journal on Computing 22, 1218-1226 (1993)

6. Schnyder, W.: Planar graphs and poset dimension. Order 5, 323-343 (1989)

7. Schnyder, W.: Embedding planar graphs on the grid. In: Proc. of the First Annual ACM-SIAM Symposium on Discrete Algorithms, pp. 138-148. SIAM, Philadelphia (1990) 